\title{
Oportunidades y obstáculos para la adaptación urbana frente al cambio climático en América Latina. Casos de la Ciudad de México, Lima y Santiago de Chile
}

Sven Schaller. Deutsches Biomasseforschungszentrum, Leipzig, Alemania. Nathalie Jean-Baptiste. Helmholtz Centre for Environmental Research, Department of Urban and Environmental Sociology, Leipzig, Alemania.

Paul Lehmann. Helmholtz Centre for Environmental Research, Department of Economics, Leipzig, Alemania.

RESUMEN | El presente artículo analiza las oportunidades y los obstáculos para la adaptación urbana al cambio climático. Se ofrece un marco analítico basado en dos grupos de variables. Primeramente, tomando en cuenta las acciones que dependen de la información, de los recursos disponibles y de los incentivos por parte de los planificadores. En segundo lugar, se considera variables vinculadas con factores secundarios, tales como las características específicas de las personas con poder de decisión, el marco institucional, y el ambiente natural y socioeconómico correspondiente. El marco analítico se aplica para examinar los casos de la Ciudad de México, Lima y Santiago de Chile. Los resultados indican que existen obstáculos similares en las tres ciudades y que el contexto institucional desempeńa un papel importante frente a los retos asociados a la integración de la adaptación, la participación y la acción del gobierno en múltiples niveles.

PALABRAS CLAVE | desarrollo urbano, gestión urbana, planificación urbana.

ABSTRACT | This paper analyzes opportunities and barriers for urban adaptation to climate change. A framework aiming at influencing decision-making was developed. Whether urban planners take action depends on three first-tier variables: information, resources, and incentives. These variables are themselves functions of underlying variables, including actor-specific characteristics of the decision-maker, the institutional environment, and the natural and socio-economic environment. The framework is applied in three case studies for Mexico City, Lima and Santiago, Chile, where anecdotal evidence has been gathered from expert interviews. The obtained results indicate that the relevant categories of barriers are mainly the same across the case studies. Moreover, the importance of the institutional context is confirmed, including challenges associated with mainstreaming adaptation, multi-level governance and participation.

KEY WORDs | urban development, urban management, urban planning.

Recibido el 9 de mayo de 2014, aprobado el 1 de noviembre de 2015

E-mail: S. Schaller, sven.schaller@dbfz.de | N. Jean-Baptiste, nathalie.jean-baptiste@ufz.de | P. Lehmann, paul.lehmann@ufz.de 


\section{Introducción}

Hoy en día, el parecer general es que los impactos resultantes del cambio climático serán más graves en las áreas urbanas que en las rurales (International Council for Local Environmental Initiatives [ICLEI], 2011), ya que las ciudades se caracterizan por una alta densidad de población, de infraestructura, y por una concentración de funciones administrativas, económicas y sociales que inciden fuertemente en el medioambiente (Bulkeley \& Betsill, 2003; Corfee-Morlot, Cochran, Hallegatte \& Teasdale, 2011; Satterthwaite, 1997). Adicionalmente, los impactos en las ciudades se exacerban por características del uso de suelo, como, por ejemplo, el alto porcentaje de superficie edificada y pavimentada, lo que resultaría en inundaciones en ocasión de lluvias intensas o prolongadas (Müller, 2012) o islas de calor (Magee, Curtis \& Wendler, 1999; Romero \& Molina, 2008). Finalmente, las ciudades presentan una fuerte dependencia de sus alrededores rurales, en especial respecto al abastecimiento de alimentos y agua potable, lo cual las hace vulnerables ante los impactos climáticos en esas regiones (Hunt \& Watkiss, 2011; McEvoy, Matczak, Banaszak \& Chorynski, 2010).

Hasta hoy, la mayoría de las acciones de adaptación al cambio climático identificadas en ciudades se han enfocado en la mitigación, la misma que se refiere, según el Grupo Intergubernamental de Expertos sobre el Cambio Climático (IPCC), a la "intervención antropógena para reducir el forzamiento antropógeno del sistema climático, [la cual] abarca diversas estrategias encaminadas a reducir las fuentes y emisiones de gases invernadero y a potenciar sus sumideros" (Parry, Canziani, Palutikof, Van der Linden \& Hanson, 2007, p. 110). Sin embargo, las emisiones de anhídrido carbónico siguen el camino del escenario más pesimista del IPCC (Manning et al., 2010), lo que implica que la temperatura global subirá más de dos grados (Joshi, Hawkins, Suttan, Lowe \& Frame, 2011). Por ello, es necesario tomar también medidas de adaptación, que, en general, representan un "ajuste de los sistemas naturales o humanos en respuesta a estímulos climáticos reales o esperados, o a sus efectos, que atenúa los efectos perjudiciales o explota las oportunidades beneficiosas" (Parry et al., 2007, p. 103). Mientras la mitigación se dirige a las causas del cambio climático, la adaptación se orienta a las consecuencias del mismo. El objetivo de las políticas de adaptación es precisamente disminuir la vulnerabilidad de los sistemas humanos y naturales relacionados al cambio climático (Fankhauser, 2009, p. 5).

En el contexto urbano, la adaptación planificada desempeña un papel clave, ya que resulta de una "decisión expresada en un marco de políticas, basada en el reconocimiento de que las condiciones han cambiado o están próximas a cambiar y de que es necesario adoptar medidas para retornar a un estado deseado, para mantenerlo o para alcanzarlo" (Parry et al., 2007, p. 103). Considerando la importancia de la urbe, la adaptación a impactos extremos del cambio climático es principalmente un desafío de planificación pública, más que una adaptación privada o autónoma (Osberghaus, Dannenberg, Mennel \& Sturm, 2010; Blanco, McCarney, Parnell, Schmidt \& Seto, 2011; Heuson et al., 2012). Por tal razón, el presente análisis se enfoca en el proceso de adaptación planificada. En particular, se analiza la preparación y adopción de estrategias y de acciones adaptativas por parte de los planificadores urbanos. 
Si bien cada vez más se considera la adaptación planificada como una acción necesaria frente al cambio climático, solamente en algunas ciudades pioneras se han elaborado estrategias en la materia (London Climate Partnership, 2006; Revi, 2008; Annals of the New York Academy of Sciences, 2010; Hardoy \& Lankao Romero, 2011; Heinrichs et al., 2011), que frecuentemente combinan medidas de adaptación con las de mitigación.

Considerando la discrepancia entre la necesidad de actuar frente al cambio climático y las iniciativas implementadas por las administraciones urbanas, sobre todo en las megaciudades de América Latina, este artículo intentará entender las oportunidades y obstáculos que puedan promover o frenar acciones adaptativas. Para ello se elaboró un esquema analítico que identifica tres determinantes clave: I) la información sobre el problema, II) los recursos disponibles y III) los incentivos para actuar. Se plantea que estos determinantes dependen, a su vez, de factores subyacentes que incluyen 1) las características específicas de los actores, 2) el marco institucional y 3) el contexto sistémico (como, por ejemplo, la ubicación geográfica o la manifestación del cambio climático).

El modelo analítico señalado se aplicó a tres megaciudades en América Latina para identificar y estructurar las oportunidades de adaptación planificada y los obstáculos a ella. Las tres ciudades estudiadas -Ciudad de México, Lima y Santiago- tienen una destacada importancia en sus respectivos países. Fueron elegidas porque son diferentes en cuanto a su ubicación, área, población, densidad y tendencias demográficas, y además tienen un distinto grado de avance en políticas de adaptación, aunque también hay similitudes entre ellas que facilitan la comparación. La heterogeneidad de estas megaciudades permitió analizar la relevancia del marco propuesto.

El esquema analítico presentado en ese artículo amplía considerablemente el debate sobre posibles obstáculos para la adaptación planificada (hay resúmenes recientes sobre la materia, como los de Eisenack \& Stecker, 2012; Biesbroek, Klostermann, Termeer \& Kabat, 2013; y Lehmann, Brenck, Gebhardt, Schaller \& Süßbauer, 2015), porque, en comparación con otras publicaciones, en él se vincula los obstáculos identificados con sus específicas razones. Un segundo aporte es que el texto no solo elabora un marco analítico, sino que brinda evidencias empíricas. Una tercera contribución es que da cuenta de los obstáculos con los que se enfrentan los planificadores urbanos en países en vías de desarrollo, en circunstancias de que solo algunos de los análisis publicados han abarcado el nivel de los tomadores locales de decisiones (Crabbé \& Robin, 2006; Amundsen, Berglund \& Westskog, 2010; Burch, 2010; Measham et al., 2011; Runhaar, Mees, Wardekker, Van der Sluijs \& Driessen, 2012) y que en su mayoría se han enfocado en la adaptación en países industrializados.

\section{Marco analítico para el análisis de oportunidades y obstáculos frente a la adaptación urbana}

El IPCC provee una categorización que distingue, por un lado, los límites físicos, ecológicos y tecnológicos a la adaptación urbana al cambio climático; y, por otro, los obstáculos financieros, cognitivos y de información, así como sociales y culturales (Adger et al., 2007). Sin embargo, tanto en el informe del IPCC como en otras 
publicaciones (véase arriba), los obstáculos se clasificaron muy a menudo de forma arbitraria, sin considerar que muchos de los mencionados están correlacionados entre sí (Corfee-Morlot et al., 2011, p. 178; Runhaar et al., 2012, p. 780). Además, las clasificaciones no siempre son explícitas o sistemáticas. En particular, no se examinan las razones tras los obstáculos. Más bien, los autores se limitan a preguntar "sí" y "cuáles" obstáculos existen, en lugar de averiguar "cómo" y "por qué" dichos obstáculos surgen (Biesbroek et al., 2013).

Considerando las críticas provenientes de estudios existentes, el marco analítico aquí presentado se enfoca justamente en el "cómo" y "por qué" surgen los obstáculos. Valdría la pena mencionar que dicho marco se basa parcialmente en el trabajo de Eisenack y Stecker (2012), así como de Moser y Ekstrom (2010). Se adaptó su metodología $a^{1}$ al distinguirse tres categorías de obstáculos, según si se vinculan a información, a recursos o a incentivos. En este enfoque, las causas generales de los obstáculos a la adaptación urbana son las características individuales de los actores, el marco institucional y el ambiente natural y socioeconómico, como se presenta en la figura siguiente.

\section{FIGURA I | El marco analítico del trabajo}

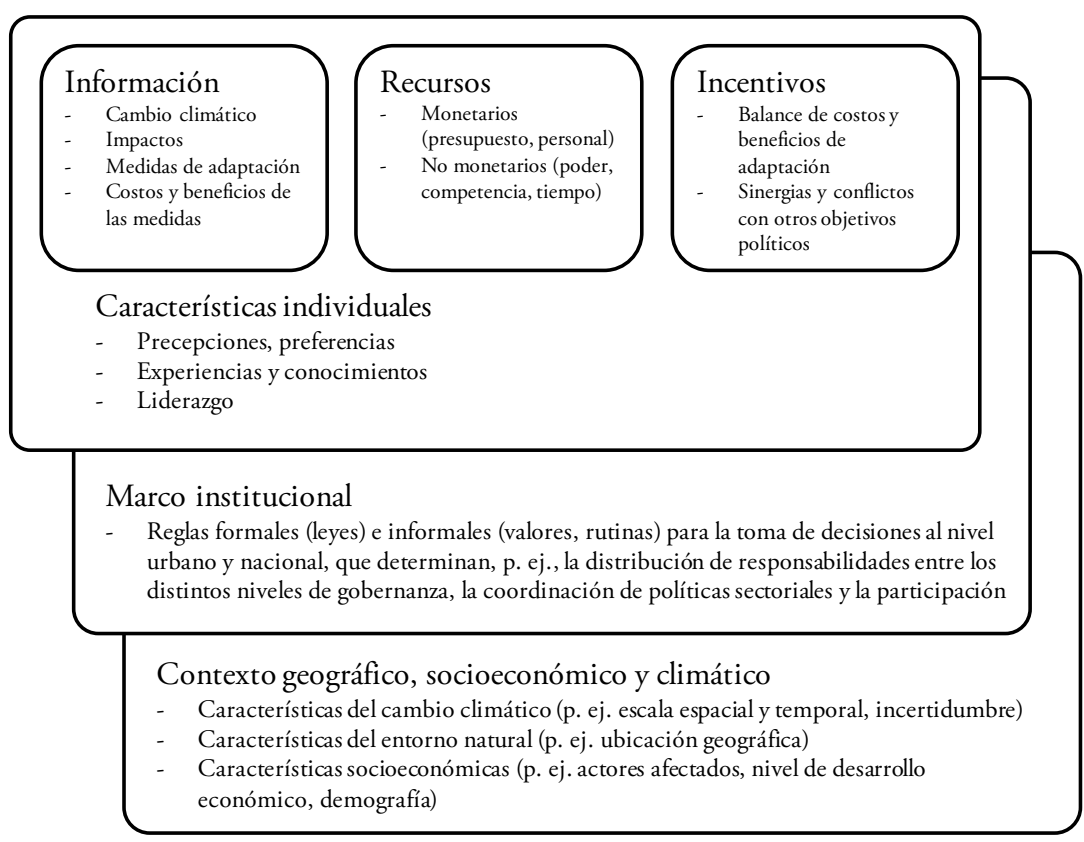

FUENTE ELABORACIÓN PROPIA

Obviamente, este marco analítico no refleja todas las interdependencias posibles entre obstáculos y oportunidades. Adicionalmente es importante destacar que dicho marco tiene un carácter principalmente descriptivo y se utilizó en el análisis

1 Para más información sobre la metodología, véase Lehmann et al. (2015). 
de las entrevistas con el fin de identificar obstáculos y oportunidades para la adaptación frente al cambio climático. Cabe mencionar que no se pretende que el nivel deseado de la planificación urbana para la adaptación se logre automáticamente con tan solo superar los impedimentos (para este argumento, véase también Moser \& Ekstrom, 2010, p. 22027).

\section{Características y adaptación planificada en las tres megaciudades}

Se realizaron estudios empíricos en tres megaciudades de América Latina: Ciudad de México, Lima y Santiago. La razón y/o justificación de que fueran elegidas apunta a una variedad de similitudes y diferencias entre ellas (véase figura 1 para sus características según las escalas del marco analítico).

Primero, estas megaciudades cobran una importancia significativa en sus respectivos países, ya que constituyen las capitales y, por lo tanto, son núcleos tanto políticos como económicos. Gran parte de la población nacional vive en ellas.

Segundo, se esperan graves impactos por el cambio climático en esas tres megaciudades. Actualmente, un desafío común para todas es la escasez de agua (Martínez López, 2011; Rehner, Samaniego \& Jordán, 2010, Comisión Nacional del Medio Ambiente [Conama, Chile], 2006). Se espera, además, que estas ciudades presenten un mayor estrés térmico (para el caso de Santiago, véase Cortés et al., 2012), lo que resultará en un aumento significativo de la cantidad de días con temperaturas extremas. Simultáneamente, se observa que debido a sus distintas ubicaciones geográficas y diferentes rasgos del cambio climático que las afectan, las ciudades en referencia deberán adaptarse a fenómenos específicos adicionales. En el caso de México, se espera una mayor frecuencia de lluvias intensas (Romero Lankao, 2010). Por su parte, el clima interanual de Lima es muy variable a causa de los fenómenos de la Oscilación del Sur-El Niño (osen), pero es muy probable que también se vea afectado por más precipitaciones e inundaciones (Consejo Nacional del Ambiente [ConAm, Perú], 1999). Las características de Santiago, en tanto, al estar ubicada en una cuenca, son sus lluvias estacionales (Cortés et al., 2012) y sus reducidas masas de aire circulante, acompańadas por fenómenos meteorológicos como la inversión térmica y la vaguada costera (Garreaud, René, Rutlland \& José, 2006).

Una tercera justificación para la selección de estas tres megaciudades remite a sus marcos institucionales. Existen grandes diferencias entre las ciudades elegidas, lo que permite una comparación del impacto de la institucionalidad sobre las oportunidades y obstáculos ante políticas de adaptación.

Cuarto, el grado de los avances en las políticas de adaptación varía considerablemente entre las tres ciudades (véase acápite "Marco institucional", en sección "Resultados"). 
TABLA I | Características de las ciudades del estudio

\begin{tabular}{|c|c|c|c|}
\hline & MÉXICO & LIMA & SANTIAGO \\
\hline \multicolumn{4}{|c|}{ Características naturales y socioeconómicas } \\
\hline $\begin{array}{l}\text { Características naturales de la } \\
\text { ubicación }\end{array}$ & $\begin{array}{l}\text { Altura, valle, clima } \\
\text { templado lluvioso }\end{array}$ & $\begin{array}{l}\text { Costa, río, clima árido, } \\
\text { fenómeno El Niño }\end{array}$ & $\begin{array}{l}\text { Cuenca, ríos, clima } \\
\text { templado-cálido }\end{array}$ \\
\hline Área & $1484 \mathrm{Km}^{2[\mathrm{~A}]^{*}}$ & $2794 \mathrm{Km}^{2[\mathrm{~A}]}$ & $840 \mathrm{Km}^{2[\mathrm{~A}]}$ \\
\hline Población & 8,9 mio. $^{[\mathrm{B}]^{* *}}$ & 9,2 mio. ${ }^{[c]}$ & 5,9 mio. ${ }^{[\mathrm{D}]}$ \\
\hline $\begin{array}{l}\text { Porcentaje de la población } \\
\text { nacional }\end{array}$ & $\begin{array}{l}8 \%[\mathrm{CDMx}] \\
18 \%[\mathrm{ZMVM}]\end{array}$ & $31 \%$ & $34 \%$ \\
\hline $\begin{array}{l}\text { PBI/cápita urbana } \\
\text { (en usD, ppp) }\end{array}$ & $17.552(2005)^{[\mathrm{A}]}$ & $7.899(2005)^{[A]}$ & $16.826(2008)^{[A]}$ \\
\hline Porcentaje del PBI nacional & $\left.17,7 \%[\mathrm{CDMx}]^{[\mathrm{E}]}\right]^{* * *}$ & $47,3 \%{ }^{[\mathrm{F}]}$ & $42,5 \%[\mathrm{G}]$ \\
\hline \multicolumn{4}{|l|}{ Características institucionales } \\
\hline $\begin{array}{l}\text { Concentración de la re- } \\
\text { caudación tributaria en los } \\
\text { gobiernos centrales }\end{array}$ & $77,8 \%{ }^{[\mathrm{н}]}$ & $98,1 \%{ }^{[\mathrm{H}]}$ & $92,6 \%{ }^{[\mathrm{H}]}$ \\
\hline Plan de adaptación urbana & Programas en vigor & $\begin{array}{l}\text { Preparación finalizada, } \\
\text { adopción pendiente }\end{array}$ & $\begin{array}{l}\text { Preparación } \\
\text { finalizada, adopción } \\
\text { pendiente }\end{array}$ \\
\hline $\begin{array}{l}\text { Año de inicio del plan de } \\
\text { adaptación }\end{array}$ & 2006 & 2011 & 2010 \\
\hline \multicolumn{4}{|c|}{$\begin{array}{l}\text { FUENTES [A]: REHNER ET AL. 2OIO; [B]: INEGI, 2OIOB; [C] INEI, 2OIOB; [D]: INE, SIN AÑO; [E]: INEGI, } \\
\text { 2OIOA; [F]: INEI, 2OIOA; [G]: BANCO CENTRAL DE CHILE, 2OO8; [H]: CETRÁNGOLO, } 2007 .\end{array}$} \\
\hline \multicolumn{4}{|c|}{ 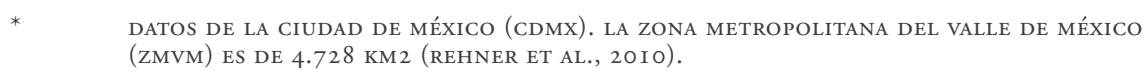 } \\
\hline \multicolumn{4}{|c|}{ ** DATOS DE LA CDMX. LA ZMVM CONTABA CON MÁS DE 20 MILLONES HABITANTES EN 20 IO. } \\
\hline
\end{tabular}

El análisis empírico presente tiene como objetivo entender hasta qué grado las características de las variables identificadas en el marco teórico han frenado o promocionado la adaptación planificada frente al cambio climático en las tres megaciudades elegidas. En particular, el énfasis se sitúa en la preparación y adopción de estrategias locales de adaptación y planes de acción por parte de los planificadores urbanos. Una breve visión de conjunto sobre el statu quo y los avances en cada una de las ciudades se da a continuación.

En México, el año 2000 marcó los inicios de algunas respuestas locales al cambio climático a través del establecimiento de la Subdirección de Cambio Climático del Gobierno del Distrito Federal. En 2004, se desarrolló la estrategia local de cambio climático que ofrece un diagnóstico sobre emisiones de gases de efecto invernadero y, además, un análisis de los riesgos de cambio climático y vulnerabilidad. Si bien esas iniciativas trataban también de la adaptación al cambio climático, se enfocaban mayormente en la mitigación. Posteriormente y dentro del marco del programa general de Desarrollo del Distrito Federal (2007-2012), del Plan Verde y de una serie de políticas públicas ambientales, el Programa de Acción Climática de la Ciudad de México (2008) surgió para dar continuidad a iniciativas preexistentes no solo con el fin de mitigar emisiones de gases de efecto invernadero, sino también para establecer varias acciones viables orientadas a diferentes actores clave (e.g., comunidades, empresas y 
gobierno local). El Programa de Acción Climática se enfoca particularmente en el cuidado del agua, manejo de microcuencas y la conservación del suelo, entre las más importantes medidas de adaptación al cambio climático. Estas acciones requirieron de una inversión de \$3 mil millones de pesos mexicanos (cerca 225 millones USD) (Secretaría del Medio Ambiente [sma, México], 2006; 2008; 2011).

La preparación de la estrategia de adaptación de Lima se inició en diciembre de 2011 a raíz del establecimiento de la Comisión Ambiental Metropolitana por el gobierno regional. Dicha comisión agrupa a representantes de los gobiernos regionales y municipales, así como a miembros del sector privado, académicos y organizaciones no gubernamentales (ONG). En el marco de la comisión se creó un grupo técnico sobre cambio climático, con el mandato de desarrollar una estrategia para la adaptación. Se invitó a participar en el grupo técnico a más expertos locales, por ejemplo del Ministerio del Ambiente y del Programa de las Naciones Unidas para el Desarrollo. La elaboración final de la estrategia se realizó gracias a contribuciones de distintas ONG, proyectos internacionales de investigación y un miembro peruano del IPCC. La fundación suiza Avina ofreció fondos adicionales para una consulta externa. La estrategia finalizó en agosto de 2012 con el apoyo de ong locales y proyectos internacionales de investigación (Municipalidad Metropolitana de Lima [MML], 2012). Se entregó al Concejo Municipal, cuya decisión final está pendiente.

Por su parte, en 2010 se empezó a elaborar un plan de adaptación para Santiago, el cual concluyó en 2012. Los principales actores involucrados fueron el Gobierno Regional y la Secretaría Regional del Ministerio del Medio Ambiente. Además, participaron en el proceso de planificación tanto las Secretarías Regionales del Ministerio de Obras Públicas, del Ministerio de Vivienda y Urbanismo y del Ministerio de Salud, como los municipios de la Región Metropolitana de Santiago. Representantes de la sociedad civil y científicos contribuyeron al proceso en el marco de mesas redondas. Sin embargo, el proceso de planificación se vio principalmente beneficiado por el apoyo de un proyecto internacional de investigación. ${ }^{2}$ El plan de adaptación debe formar parte del Plan Regional de Desarrollo de Santiago que será aprobado por el Gobierno Regional (conama, 2008).

\section{Métodos}

Se realizaron entrevistas con funcionarios administrativos, políticos regionales, científicos y representantes de ONG (véase tabla 2). Se eligió a los entrevistados por estar directamente incluidos en la planificación de políticas adaptativas o porque fueron referenciados por otros expertos. Para garantizar que se pudiese comparar el marco institucional de las tres ciudades, los expertos entrevistados debían tener

2 Clima Adaptación Santiago (CAS). Adaptación al cambio climático en megaciudades de América Latina. Red Regional de Aprendizaje. Documento de proyecto Cepal [LC/W.529 - 2013-95]. Editado por K. Krellenber, R. Jordán, J. Rehner, A. Schwarz, B. Infante, K. Barth y A. Pérez. Santiago: Comisión Económica para América Latina y el Caribe (CEPAL) / Ministerio Federal de Cooperación Económica y Desarrollo, Alemania / Helmholtz Zentrum für Umweltforschung (Ufz), Leipzig, Alemania, 2013. Disponible en http://www.cepal.org/es/publicaciones/4056clima-adaptacion-santiago-cas-adaptacion-al-cambio-climatico-megaciudades-america 
puestos administrativos similares y cierta posición de responsabilidad en aquellas funciones. En total se realizaron 23 entrevistas.

En cada ciudad se utilizaron preguntas predeterminadas y orientadas a las categorías principales del marco analítico (véase tabla 3). La información sobre factores subyacentes -es decir, las características de los actores, del marco institucional y del ambiente natural y socioeconómico- se obtuvo en forma indirecta o por análisis de documentos.

TABLA 2 Sinopsis de las entrevistas

\begin{tabular}{|c|c|c|c|}
\hline CIUDAD & ENTREVISTADO & CóDIGo & FECHA \\
\hline \multirow{5}{*}{ México } & Empleado de Gobierno & M01 & 10 noviembre 2011 \\
\hline & Investigador & M02 & 21 marzo 2012 \\
\hline & Empleado de Gobierno & M03 & 20 abril 2012 \\
\hline & Empleado de Gobierno & M04 & 20 abril 2012 \\
\hline & $\begin{array}{l}\text { Miembro de una Organización no Gubernamental } \\
\text { (oNG) }\end{array}$ & M05 & 28 noviembre 2013 \\
\hline \multirow{11}{*}{ Lima } & Empleado de un Ministerio Público & L01 & 19 octubre 2011 \\
\hline & Empleado de un Ministerio Público & L02 & 19 octubre 2011 \\
\hline & Empleado de un Ministerio Público & L03 & 20 octubre 2011 \\
\hline & Empleado de un Ministerio Público & L04 & 20 octubre 2011 \\
\hline & Empleado de un Ministerio Público & L05 & 20 octubre 2011 \\
\hline & Empleado de Gobierno & L06 & 20 octubre 2011 \\
\hline & Investigador & L07 & 24 octubre 2011 \\
\hline & Investigador & L08 & 21 octubre 2011 \\
\hline & Miembro de una ONG & L09 & 17 octubre 2011 \\
\hline & Miembro de una ONG & L10 & 21 octubre 2011 \\
\hline & Miembro de una oNG internacional & L11 & 24 octubre 2011 \\
\hline \multirow{7}{*}{ Santiago } & Empleado de un Ministerio Público & S01 & 18 enero 2012 \\
\hline & Empleado de un Ministerio Público & S02 & 24 enero 2012 \\
\hline & Empleado de un Ministerio Público & S03 & 27 enero 2012 \\
\hline & Empleado de un Ministerio Público & S04 & 27 enero 2012 \\
\hline & Empleado de una Oficina de Gobierno & S05 & 23 enero 2012 \\
\hline & Investigador & S06 & 18 enero 2012 \\
\hline & Miembro de una ONG & S07 & 24 enero 2012 \\
\hline
\end{tabular}

FUENTE ELABORACIÓN PROPIA 
TABLA 3 | Guía para las entrevistas

\begin{tabular}{|c|c|}
\hline \multicolumn{2}{|c|}{ INFORMACIÓN } \\
\hline 1.1 & $\begin{array}{l}\text { ¿Qué información existe sobre el cambio climático y los impactos en su ciudad? ¿Quién tiene } \\
\text { acceso a la información sobre el cambio climático y los impactos? }\end{array}$ \\
\hline 1.2 & $\begin{array}{l}\text { ¿Qué información considera Ud. necesaria para que las instituciones puedan reaccionar política- } \\
\text { mente? ¿Cuáles han sido los principales obstáculos para obtener esa información? ¿Qué medidas } \\
\text { concretas de adaptación conoce Ud. a nivel local? }\end{array}$ \\
\hline 1.3 & ¿Qué medidas de adaptación (de corto y largo plazo) considera Ud. importantes? \\
\hline 1.4 & $\begin{array}{l}\text { ¿Qué medidas de adaptación han sido implementadas en su ciudad? ¿Qué medidas concretas de } \\
\text { adaptación han sido planificadas? ¿Cuáles han sido los principales obstáculos en este contexto? }\end{array}$ \\
\hline 1.5 & ¿Existen montos estimados de los costos y beneficios de la adaptación a nivel metropolitano? \\
\hline \multicolumn{2}{|c|}{ RECURSOS } \\
\hline 2.1 & $\begin{array}{l}\text { ¿Cuáles son las prioridades que contempla el gasto público actual? ¿Cuál es el presupuesto que } \\
\text { se tiene para cada uno de los objetivos de desarrollo urbano? ¿Cuál es el presupuesto que se tiene } \\
\text { para la adaptación en su ciudad? ¿Este presupuesto se aumentará en los próximos cinco años? } \\
\text { ¿Qué porcentaje del presupuesto total representa el presupuesto para la adaptación? }\end{array}$ \\
\hline 2.2 & $\begin{array}{l}\text { ¿Hay un financiamiento especial a nivel metropolitano para la adaptación? ¿Utiliza Ud. fondos } \\
\text { nacionales o internacionales? }\end{array}$ \\
\hline 2.3 & $\begin{array}{l}\text { ¿Qué institución en su ciudad es responsable por la planeación de medidas de adaptación? ¿Exis- } \\
\text { ten varias instituciones responsables? ¿Cuál es el organismo a nivel metropolitano que coordina } \\
\text { estas actividades? ¿Cómo lo hace? }\end{array}$ \\
\hline 2.4 & $\begin{array}{l}\text { ¿Qué actores participan en la implementación de medidas de adaptación? ¿Cómo ve Ud. los } \\
\text { recursos de personal disponibles en su ciudad? }\end{array}$ \\
\hline \multicolumn{2}{|c|}{ INCENTIVOS } \\
\hline 3.1 & $\begin{array}{l}\text { ¿Cuál es el objetivo de desarrollo urbano que Ud. persigue cuando planea o implementa medidas } \\
\text { de adaptación frente al cambio climático? }\end{array}$ \\
\hline 3.2 & $\begin{array}{l}\text { En su opinión, ¿cuáles son los objetivos más importantes en la política urbana? ¿Qué impor- } \\
\text { tancia tiene el cambio climático y la adaptación a los impactos del cambio climático en este } \\
\text { contexto? }\end{array}$ \\
\hline 3.3 & $\begin{array}{l}\text { ¿Cuáles son los objetivos que impiden la adaptación a los impactos del cambio climático? ¿Exis- } \\
\text { ten objetivos contradictorios? }\end{array}$ \\
\hline 3.4 & ¿Qué actores impulsan la implementación de medidas de adaptación? ¿De qué manera? \\
\hline 3.5 & $\begin{array}{l}\text { ¿Cuáles son los riegos del cambio climático que tienen influencia en la política de adaptación } \\
\text { frente a este fenómeno? }\end{array}$ \\
\hline
\end{tabular}

FUENTE ELABORACIÓN PROPIA

Para el análisis, se transcribieron las entrevistas, se agruparon las declaraciones de acuerdo con las variables del marco analítico, se parafraseó el contenido y se generalizaron las glosas resultantes para formular categorías abstractas. La comparación de las diferentes categorías permitió identificar obstáculos y oportunidades específicos de cada ciudad. Por último, se agruparon y compararon los resultados de las tres ciudades, obteniéndose obstáculos y oportunidades generales. Siendo un estudio cualitativo, los resultados no pretenden ser representativos. Más bien dan evidencias puntuales que podrían abrir camino para estudios más amplios sobre la adaptación urbana planificada.

Los resultados empíricos desarrollados a continuación se establecieron con referencia a las variables principales (información, recursos e incentivos) y en función a los factores subyacentes (características individuales de los actores, ambiente institucional, y contexto geográfico, socioeconómico y climático) del marco analítico. Las declaraciones de los expertos están indicadas por códigos (véase tabla 2). 


\section{Resultados}

\section{Información - características individuales de los actores}

En las entrevistas se identificaron factores que influyen en la información que reciben los tomadores de decisiones. En Lima, los entrevistados repetidamente reportaron que tanto la población como los tomadores de decisiones administrativas no han desarrollado conciencia respecto de la posible magnitud del cambio climático y, por lo tanto, perciben los posibles impactos como algo muy lejano en el futuro (L03, L05, L09, L10). En Santiago, los expertos mencionaron percepciones similares entre los actores políticos y los planificadores urbanos o regionales. Además, se considera el cambio climático como un tema que pertenece más a la ciencia (S01, S05) que a la política o planificación. Como consecuencia, muchos tomadores de decisiones, sobre todo a nivel nacional, no saben distinguir entre la mitigación y la adaptación (S07). Por otra parte, a los actores locales les falta muy a menudo el acceso a información sobre el cambio climático y sus impactos (S01, S07). En México, se señaló la dificultad de concientizar a los funcionarios públicos respecto de la importancia de considerar el cambio climático dentro del diseño de políticas públicas (M01, M05). Esa barrera fue mencionada como uno de los mayores retos para implementar medidas de adaptación en diferentes sectores. Adicionalmente, se reveló la necesidad de los gobiernos locales de conocer los escenarios climáticos para tomar mejores decisiones a largo plazo (M03). Finalmente, las entrevistas indicaron una diferencia del interés por obtener información entre sectores (M02, M03, M04, M05); en efecto, las disposiciones frente a las políticas de adaptación varían de un sector al otro, en función de la voluntad política, sinergia entre sectores y el uso de la información.

Un aspecto determinante para el acceso y uso de información se encuentra en el interés personal de los tomadores de decisiones y su involucramiento en el tema. Este compromiso es importante para actuar de forma proactiva y para establecer y mantener contactos personales con otros expertos administrativos o externos. Eso se nota especialmente en Lima, donde después del cambio de gobierno en 2011, muchos activistas de ONG ambientales se incorporaron a los ministerios y a la administración municipal. Estos actores, debido a su quehacer previo, demostraron sus preferencias por la protección del medioambiente e impulsaron acciones concretas, a pesar del déficit de información y de la incertidumbre relacionada con los impactos del cambio climático (L09, L10). Por un lado, este interés ambiental podría ser interpretado como una oportunidad para iniciar acciones de adaptación. Por otro, al omitir dichas incertidumbres, se corre el riesgo de un sobreajuste o una mala adaptación, lo que, a su vez, constituye un obstáculo para establecer un nivel adecuado de adaptación (L07).

\section{Información - marco institucional}

El obstáculo más importante mencionado para Lima es la falta de una organización coordinadora y la cooperación limitada entre las organizaciones. Si bien algunos actores tienen acceso a información relevante, solo colaboran en aspectos específicos (L06). Eso podría ser considerado como la razón principal por la cual falta un mainstreaming de adaptación, esto es, la integración de la adaptación a políticas 
sectoriales. Adicionalmente, carecen de una mejor comunicación interna y un "lenguaje común” para el desarrollo de una estrategia general de adaptación (L06). De igual manera, los entrevistados en Santiago consideraron como uno de los principales obstáculos la falta de una organización coordinadora que proporcione regularmente información actualizada (S02, S04, S05, S07). En contraste, en México existe desde hace una década la Secretaría del Medio Ambiente del Distrito Federal (SEDEMA), que busca integrar la calidad del aire y el cambio climático entre sus seis rubros prioritarios ambientales en la Ciudad de México. La sedema representa el organismo impulsor a través del Programa de Cambio Climático (M01, M03). Sin embargo, su influencia no deja de ser limitada, ya que su jurisdicción se limita a la Ciudad de México y no abarca la Zona Metropolitana del Valle de México.

En todas las ciudades analizadas, los múltiples niveles de gobierno resultan inadecuados en relación con los mecanismos para generar y distribuir datos relevantes sobre el cambio climático. En Perú, el Gobierno Nacional es muy reservado para invertir en la captura, suministro y distribución de información, lo cual podría mejorar la base de datos existente para decisiones locales (L03, L07). Además, las organizaciones clave no están incluidas en los pocos proyectos de investigación. Por ende, el intercambio de información entre los expertos locales depende principalmente de reuniones informales (L08). Respecto a Santiago, se inició un diálogo entre la ciencia, la política y la administración. Sin embargo, todavía falta una plataforma permanente para institucionalizar el intercambio (S05). Además, existen canales administrativos múltiples y complejos que llevan a una demora en la distribución (o pérdida) de información (S02). En México, si bien existen varias instituciones que tratan el tema, la difusión de la información sobre el cambio climático a nivel local se da de manera informal. A escala federal, el discurso oficial se genera a través de la Secretaría de Medio Ambiente y Recursos Naturales, que define las pautas y programas operativos aplicables provenientes de otros organismos federales (M02). En las entrevistas se señaló el carácter informal de los intercambios. Al respecto, un participante mencionó que las discusiones clave, las decisiones o impasses se resuelven a través de encuentros breves y "cabildeo constante" (M01).

Los entrevistados se refirieron a falta de memoria institucional debido a una rotación significativa de los empleados en la administración pública durante los cambios de gobierno. Bajo tales circunstancias, se vuelve difícil asegurar una planificación a largo plazo, ya que se debe capacitar constantemente al personal en el puesto (L05, S01, S04, S07). Por eso, proyectos externos y/u ong brindan a menudo la información que normalmente debería venir de las instituciones públicas (L11).

\section{Información - contexto geográfico, socioeconómico y climático}

En todas las ciudades analizadas, la información científica sobre el cambio climático a nivel regional no está disponible aún o está caracterizada por un porcentaje alto de incertidumbre a nivel local. Esta situación se debe a la falta general de conocimientos acerca de modelos de simulación climática y del downscaling, es decir, la reducción de escala de modelos globales. Esta falta representa, en primer lugar, una consecuencia de la complejidad del ambiente natural. Además, el estatus de desarrollo de los países desempeńa un papel importante en este contexto, ya que se 
implica también las capacidades científicas. En Santiago, por ejemplo, los expertos mencionaron que es difícil elaborar un plan de adaptación con las estimaciones existentes y que es necesario bajar la escala de los datos regionales disponibles (S04, S07). También en Lima, un impedimento importante es la falta de datos de proyección en alta resolución y el desarrollo impredecible del fenómeno Oscilación del Sur-El Niño (osen), que representa un desafío adicional para el pronóstico de los impactos locales del cambio climático en esta ciudad (L07). Por tal razón, la información sobre los impactos climáticos es considerada como difusa, dispersa o simplemente no disponible, lo que impide elaborar un plan de adaptación local (L06). Obstáculos similares fueron reportados en México, sobre todo con respecto al acceso a datos confiables y a la capacidad de las entidades públicas de manejar dicha información. Se habló en particular de la dificultad de mantenerse actualizados, ya que los datos de proyección global se generan más rápido que la capacidad para manejarlos a nivel local (M01, M03).

\section{Recursos - características individuales de los actores}

En las tres ciudades las características individuales de los actores no parecen influenciar la disponibilidad de los recursos, según las personas entrevistadas.

\section{Recursos - marco institucional}

En general, en las tres ciudades surge el problema del mainstreaming, es decir, la integración y el fomento de la adaptación en diferentes sectores y gremios. Por lo tanto, el acceso a recursos está severamente limitado. En Santiago, por ejemplo, aún no existe ninguna mención para actividades relacionadas con la mitigación o adaptación en el presupuesto general de los gobiernos nacionales y locales (S05, S06).

La razón tras el fracaso del mainstreaming está en una coordinación inapropiada entre los sectores (M01, M02, M04, L08, S02, S04, S05, S07). En ciudades donde se estableció una organización coordinadora, como es el caso de Lima, se han concentrado distintos departamentos municipales y regionales, con el fin de guiar las acciones de adaptación a nivel local (L06). Sin embargo, darle la responsabilidad a una entidad del medioambiente implica que la planificación de la adaptación está estructurada y considerada primeramente como un asunto ambiental y, consecuentemente, recibe poco apoyo político (L03, L06). También en México, la adaptación está vista en gran parte desde una perspectiva ambiental. En la Ciudad de México, la institución coordinadora está relacionada con la Secretaría del Medio Ambiente. Hay que destacar que el problema de la falta de recursos se ha recrudecido en diversos y múltiples niveles por diferentes motivos ligados al ámbito gubernamental. Por ejemplo, la carencia financiera de la administración pública en Santiago se atribuye al gobierno nacional, que desarrolla una política de mercado cuyas características son un bajo porcentaje de gastos públicos y un marco regulatorio mínimo (S01, S02, S07). Además, no existiendo un federalismo fiscal en Chile, cualquier cumplimiento de políticas depende de un Fondo Común Municipal (S05, S06, S07). La coincidencia parcial y la competencia entre varios niveles administrativos resulta en una planificación de adaptación tanto en Lima como en Santiago, que consume muchos recursos, ya que una parte significativa de recursos humanos y financieros debe ser 
invertida en la coordinación de las actividades de los diferentes actores (L05, L08, L10, L11, S02, S07). En cuanto a México, si bien existe una entidad coordinadora, los recursos humanos para dicha área no son suficientes. La Dirección de Cambio Climático, por ejemplo, cuenta con cinco empleados, quienes se encargan de establecer los lineamientos que se deben seguir y buscan apoyo de diversas entidades/ sectores para implementar ciertas medidas, particularmente en la conservación del agua y reforestación urbana y rural (M01). En el sector de la vivienda se mencionó, además de la limitante financiera, la falta de continuidad de los proyectos existentes (M04).

\section{Recursos - contexto geográfico, socioeconómico y climático}

Aparte de los aspectos institucionales, se puede atribuir la falta de recursos humanos y financieros a las características socioeconómicas de los países. Siendo estas decisivas para la disponibilidad de fondos públicos, se tiene que lo socioeconómico influye en el nivel de importancia de la adaptación. De forma general, los escasos recursos públicos se distribuyen según fines políticos y la disponibilidad de recursos está estrechamente vinculada con promesas. Por ejemplo, en Perú la reducción de la pobreza y la adaptación en las regiones rurales tienen preferencia dentro de las políticas nacionales (L03, L06, L07, L10). Por tal razón, existen pocos fondos para financiar medidas de adaptación en centros urbanos. El apoyo financiero se da a través de proyectos piloto escogidos por parte de organizaciones internacionales (L06, L09, L10). En general, se puede observar que los temas ambientales a nivel local tienen en Lima una importancia subordinada. Los recursos financieros del Departamento del Medio Ambiente constituyen solo el 3\% del presupuesto municipal (L06). En Chile se utilizan escasos recursos públicos en el tema, que son asignados en primer lugar a promover actividades en los sectores de la exportación, en vez de financiar medidas de adaptación urbana (S07). En México, la adaptación urbana al cambio climático si bien no desempeña un papel dominante en el presupuesto municipal, llega a filtrarse de alguna forma dentro de sectores como los de la salud, manejo de agua y transporte. La prioridad de la política urbana se determina por el plan de desarrollo urbano, normalmente sexenal, y por los diferentes programas que se desprenden de ese plan maestro. Es a través de dicho plan que ciertas acciones de adaptación se llegan a implementar (M01).

\section{Incentivos- características individuales de los actores}

La actitud de los tomadores de decisiones es un determinante importante para la planificación de la adaptación en todas las ciudades. En Chile, los entrevistados atribuyeron poca iniciativa a las personas con poder de decisión, y lamentan la falta de voluntad para asumir cierta responsabilidad y hacer compromisos políticos ( $\mathrm{S} 01$, S04, S05). La Ciudad de México se caracteriza por tener un gobierno local comprometido con el medioambiente y la temática del cambio climático. Ciertos tomadores de decisiones han asumido un papel particularmente activo tanto a escala local como internacional. En la Secretaría del Medio Ambiente hay actores con poder de influencia a altos niveles (M01). De manera similar, en Lima la función de ciertos actores constituye un factor estimulante para la planificación de adaptación. Actores 
que tiempo atrás tuvieron la responsabilidad de desarrollar una estrategia local de adaptación y un plan de acción y quienes trabajaron en diferentes ONG ambientales, demostraron un compromiso especial con la temática (L06, L08, L10).

\section{Incentivos - marco institucional}

Un obstáculo importante es la carencia de un mandato para la planificación de la adaptación por parte de las autoridades municipales. En Chile, por ejemplo, no hay normas legales e instrumentos para guiar a la autoridad local (S01, S04, S07). Un empleado de un ministerio atribuye esto al enfoque del gobierno neoliberal de Chile; él personalmente prefiere soluciones provenientes de una intervención estatal (S02). Una notable excepción en términos de orientación nacional está en Perú, donde existe una ley nacional que obliga a las autoridades regionales a adoptar una estrategia de adaptación y un plan de acción (L09, L10).

La falta de coordinación entre las administraciones urbanas y rurales, así como entre las regiones y estados, se plantea como un problema específico del gobierno a múltiples niveles en el Perú, Chile y México (M01, M03, L08, S02, S07). Este déficit dificulta la aplicación de medidas de adaptación más allá de fronteras administrativas, lo que puede ser especialmente perjudicial para desafíos a mayor escala, como los impactos sobre el abastecimiento de agua.

Un factor que ha sido un motor para la toma de decisiones en la adaptación es el apoyo financiero de varias organizaciones internacionales para enfrentar la temática climática; de hecho, como requisito para sus aportes, esas organizaciones plantean la adopción de políticas climáticas locales. En el Perú, por ejemplo, existen fondos similares aportados por la cooperación al desarrollo (L09).

En Lima y Santiago, los expertos de los ministerios y de las ong se quejaron de la burocracia que dificulta la integración de la adaptación (L09, S01, S02). En Lima, los empleados a menudo se niegan a asumir responsabilidades adicionales y simplemente no consideran acciones de adaptación. Este hecho se ve agravado debido a que los salarios de los empleados no se basan en resultados, lo cual reduce los incentivos para actuar (L06, L10).

En México, se habla de la necesidad de una coordinación metropolitana que abarcaría la Ciudad de México, el Gobierno del Estado de México y municipios vecinos. Se lamenta también la falta de voluntad política por parte de municipios dirigidos por partidos opuestos, que frenan así la toma de decisiones comunes y acciones conjuntas (M03). De igual manera, en Chile se argumentó que los largos procedimientos administrativos para la aprobación de las medidas de adaptación pueden llegar distraer a los responsables políticos y, consecuentemente, retrasar acciones de adaptación (S02).

\section{Incentivos - contexto geográfico, socioeconómico y climático}

En las tres ciudades, el contexto geográfico, socioeconómico y climático parece influenciar la adaptación planificada. En primer lugar, el conocimiento limitado de los impactos del cambio climático perjudica los incentivos para actuar. Eso se debe a tres factores. Primero la complejidad del sistema climático, pues complica el entendimiento del contexto urbano y de los beneficios de la adaptación. Segundo, 
particularmente en el caso de Lima el cambio climático no se observa continuamente sino más bien a partir de grandes irregularidades (fenómeno osEn cada 5 a 10 años) (L07). Tercero, muchos beneficios de adaptación planificada se materializan solamente a largo plazo. Este hecho está en contradicción con los cortos plazos políticos y la rotación resultante de los empleados administrativos (L08, L10). Por consecuencia, existen pocos incentivos para dirigir esfuerzos de planeación hacia la adaptación al cambio climático (L06, L10, S02). Ello es de suma importancia, porque las condiciones socioeconómicas implican acciones con resultados a corto plazo en otros campos políticamente priorizados, por ejemplo, la seguridad pública (M01, M03, L09, S01, S02).

\section{Discusión}

En la tabla 4 ofrecemos un resumen de los resultados empíricos. Si bien la evidencia del presente estudio es puntual, es posible plantear algunos resultados globales.

\section{TABLA 4 Resumen de los resultados empíricos}

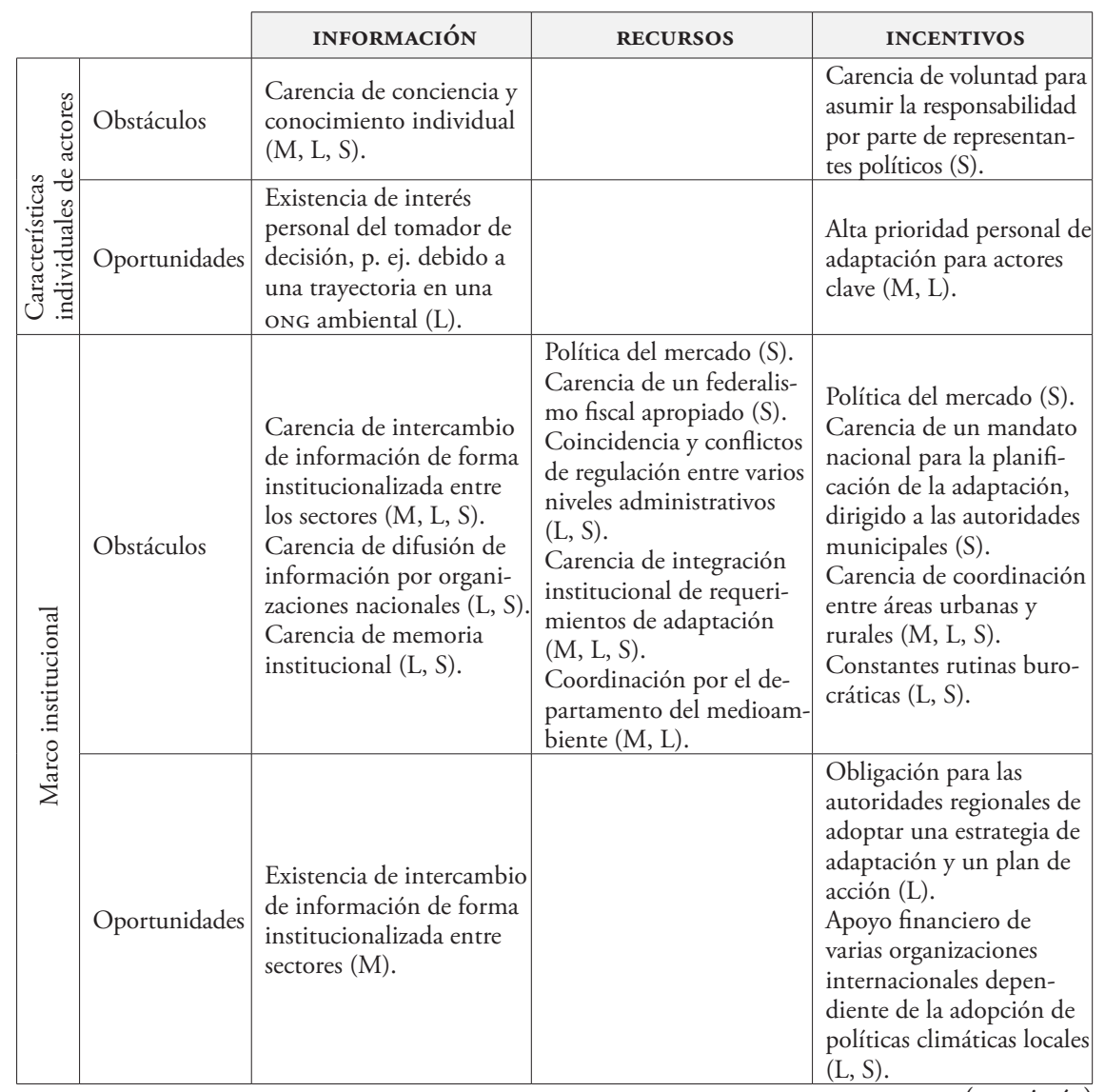


(continuación)

\begin{tabular}{|c|c|c|c|c|}
\hline & INFORMACIÓN & RECURSOS & INCENTIVOS \\
\hline \multirow{2}{*}{ 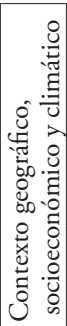 } & Obstáculos & $\begin{array}{l}\text { Complejidad del ambien- } \\
\text { te natural }(\mathrm{M}, \mathrm{L}, \mathrm{S}) \text {. } \\
\text { Bajo nivel de desarrollo } \\
\text { económico que perjudica } \\
\text { capacidades científicas } \\
(\mathrm{M}, \mathrm{L}) \text {. }\end{array}$ & $\begin{array}{l}\text { Carencia de presupuesto } \\
\text { por bajo nivel de desarro- } \\
\text { llo }(\mathrm{L}, \mathrm{S}) \text {. } \\
\text { Alta prioridad de otros } \\
\text { objetivos públicos }(\mathrm{L}, \mathrm{S}) \text {. }\end{array}$ & $\begin{array}{l}\text { La complejidad y el lapso } \\
\text { del cambio climático } \\
\text { impiden incentivos de } \\
\text { acción (L, S). } \\
\text { Incentivos dirigidos a } \\
\text { otros objetivos políticos } \\
(\mathrm{M}, \mathrm{L}, \mathrm{S}) \text {. }\end{array}$ \\
\hline & Oportunidades & & & \\
\hline
\end{tabular}

LEYENDA L - LIMA, S - SANTIAGO, M - MÉXICO

NOTA CASILLAS VACÍAS INDICAN QUE NO SE IDENTIFICARON OBSTÁCULOS/OPORTUNIDADES ESPECÍFICOS EN LAS ENTREVISTAS. ESTO NO IMPLICA QUE TALES OBSTÁCULOS/OPORTUNIDADES ESTÉN AUSENTES EN LAS CIUDADES EN GENERAL.

En primer lugar, nuestros resultados empíricos confirman algunos factores institucionales mencionados en la literatura: un desafío importante en todas las ciudades es el mainstreaming de la adaptación. Muchos de los entrevistados confirmaron que la planificación de la adaptación no debería ser un asunto ambiental, sino estar incluido en los procedimientos sectoriales existentes, como señala la literatura (Füssel, 2007; Adger et al., 2009; Measham et al., 2011; United Nations Development Programme/United Nations Environment Programme [undp/unep], 2011). Por otro lado, se argumentó que el mainstreaming todavía no se implementa en las ciudades y que se carece de una coordinación transversal necesaria para una efectiva implementación (Hunt \& Watkiss, 2011). En este contexto, una asignación de coordinación dentro de un departamento o ministerio ambiental podría dificultar dicho mainstreaming, ya que se podría considerar la adaptación primeramente como un asunto ambiental (Measham et al., 2011; undp/unep, 2011). En relación con los diferentes niveles de gobierno, nuestros resultados sugieren que una dirección (para mejorar la información), un apoyo financiero (para superar las restricciones de recursos) y un mandato (para poner incentivos apropiados) por parte del gobierno nacional son aspectos esenciales para la planificación local de la adaptación. La ausencia de tal marco nacional se observa a menudo en la literatura como un obstáculo clave (Koch, Vogel \& Patel, 2007; Amundsen et al., 2010; Burch, 2010; Corfee-Morlot et al., 2011). Las rutinas y procedimientos burocráticos en las tres ciudades son obstáculos importantes para la adaptación. La participación está gradualmente ganando importancia en las tres ciudades y eso podría ayudar a sobrepasar las restricciones en cuanto a la información y recursos (véase también Adger et al., 2009; Corfee-Morlot et al., 2011; Hunt \& Watkiss, 2011). La experiencia indica que se han llevado a cabo algunos pasos iniciales, como, por ejemplo, la colaboración entre la ciencia y las ONG. Sin embargo, todavía se carece de la participación permanente e institucionalizada de la sociedad civil.

Segundo, el análisis presente confirma que existen obstáculos y oportunidades particulares a la adaptación planificada: la complejidad del contexto natural conlleva grandes incertidumbres en relación con los impactos del cambio climático. Resulta difícil especificar objetivos y resulta igualmente difícil calcular la efectividad de las 
medidas de adaptación para cumplir con dichos objetivos. Esta dificultad claramente perjudica los incentivos para invertir en la planificación de la adaptación (véase también Biesbroek et al., 2013). Por otro lado, hay una obvia discordancia entre - por un lado- el largo plazo de los impactos climáticos y de los efectos de las medidas de adaptación; y -por otro- el relativamente corto plazo de las elecciones y procedimientos municipales para la planeación de la adaptación. Esta discordancia causa otro obstáculo importante, como es la limitante económica por parte de los planificadores urbanos. Los costos de la planificación adaptativa surgen a corto plazo, mientras que los beneficios de la adaptación se materializan normalmente a largo plazo (véase también Biesbroek et al., 2013). Además, la adaptación es una tarea transversal de planificación, cuyos co-beneficios son decisivos para tomar decisiones políticas, crear incentivos para actuar y para liberar fondos.

Tercero, algunos de los obstáculos identificados son problemas específicos de toma de decisiones a nivel local, regional y nacional.

Muchas decisiones locales de la planificación adaptativa dependen del marco institucional de los niveles administrativos superiores. En este contexto, un mandato explícito nacional para adoptar estrategias locales y para transferir recursos, podría mejorar considerablemente las oportunidades para la planificación de la adaptación en las ciudades (véase también el párrafo sobre la gobernanza de múltiples niveles).

Las ciudades no solo son vulnerables a los impactos climáticos en su territorio, sino también en su entorno. Desde un punto de vista institucional, la adaptación a estos impactos está fuera del ámbito de la planeación adaptativa urbana. Por eso, podría haber una discordancia espacial entre el ambiente natural y el institucional. Un marco apropiado de gobernanza necesitaría, en consecuencia, un mecanismo de coordinación entre las áreas urbanas y rurales (Moser \& Ekstrom, 2010, p. 22028).

\section{Conclusiones}

Este artículo examinó obstáculos y oportunidades para la planificación urbana de adaptación por tomadores de decisiones municipales. El foco particular de nuestro análisis ha estado en el proceso de preparación y adopción de estrategias de adaptación urbana y de planes de acción. Para este objetivo, se propuso un marco teórico en el cual se distingue diferentes variables, que podrían constituir obstáculos y oportunidades para la planificación de la adaptación.

La innovación principal de este marco teórico está en su representación explícita de interacciones entre diferentes categorías de obstáculos (y oportunidades) que se discuten frecuentemente en la literatura, pero muy a menudo de forma separada. Con lo anterior, el texto contribuye a un mejor entendimiento de cómo surgen los obstáculos y cómo podrían ser superados. Se utiliza el marco teórico para proveer y analizar evidencia empírica en cuanto a la existencia y relevancia de diferentes obstáculos y oportunidades en tres ciudades de Latinoamérica: Lima (Perú), Santiago de Chile y Ciudad de México.

Pese a la heterogeneidad de las ciudades, todos los obstáculos y oportunidades que mencionaron los tomadores de decisiones se pueden atribuir a las variables especificadas del marco teórico presente. Por eso, un resultado del análisis podría ser 
que dicho marco se puede aplicar en diferentes ciudades del mundo para explicar ciertos procesos de planificación de la adaptación.

Además, el marco teórico podría ser un punto de inicio de recomendaciones políticas para superar obstáculos relacionados con la planificación de la adaptación, lo que correspondería a un subsecuente paso analítico después del análisis de los obstáculos. El marco teórico ayuda a desentrañar hasta qué grado las características de las variables principales (información, recursos, incentivos) dependen de uno o más factores subyacentes (características individuales de los actores; marco institucional; contexto geográfico, socioeconómico y climático). Nuestro análisis muestra que tres opciones institucionales pueden promover de manera efectiva la adaptación al cambio climático: a) el mainstreaming de la adaptación en todos los sectores urbanos, lo que permite destacar co-beneficios de adaptación; b) un mandato explícito desde el nivel nacional para la adopción de planes de adaptación al nivel regional o municipal; y c) la participación de los distintos actores sociales en la toma de decisiones.

Desde luego, existen preguntas científicas todavía abiertas en relación con los obstáculos para la adaptación al cambio climático. Primero, hay que aprender más sobre las causas de dichos obstáculos y oportunidades, utilizando nuevas estrategias para entender la actitud humana (véase, por ejemplo, la contribución económica en Gawel, Heuson \& Lehmann, 2012). Segundo, se necesita mayor comprensión de la relevancia de los distintos obstáculos y sus orígenes. Sería útil partir de la evidencia puntual (ya existente en varios estudios, incluido el presente) y realizar, de ser posible, más estudios cuantitativos. Por último, sería de mayor ayuda si se pudiese cambiar de un análisis de obstáculos hacia uno de soluciones. El primero, indudablemente, es el prerrequisito para lo segundo.

\section{Referencias bibliográficas}

Adger, W. N., Agrawala, S., Mirza, M. M. Q., Conde, C., O’Brien, K., Pulhin, J. \& Takahashi, K. (2007). Assessment of adaptation practices, options, constraints and capacity. En M. L. Parry, O. F. Canziani, J. P. Palutikof, P. J. van der Linden \& C. E. Hanson (Eds.), Climate change 2007: Impacts, adaptation and vulnerability. Contribution of Working Group II to the Fourth Assessment Report of the Intergovernmental Panel on Climate Change (pp. 717-743). Cambridge: Cambridge University Press.

Adger, W. N., Dessai, S., Goulden, M., Hulme, M., Lorenzoni, I., Nelson, D. R. \& Wreford, A. (2009). Are there social limits to adaptation to climate change? Climatic Change, 93 (3-4), 335-354. doi: 10.1007/s10584-008-9520-z

Amundsen, H., Berglund, F. \& Westskog, H. (2010). Overcoming barriers to climate change adaptation - A question of multilevel governance? Environment and Planning C: Governance and Policy, 28 (2), 276-289. doi: 0.1068/c0941

Annals of the New York Academy of Sciences (2010). Climate change adaptation in New York City. Building a risk management response. New York: New York Academy of Sciences. 
Banco Central de Chile. (2008). Ficha: Producto Interno Bruto Regional 2003-2006, Base 2003. Consultado 12 de marzo de 2014. En http://www.bcentral.cl/publicaciones/ estadisticas/actividad-economica-gasto/aeg07b.htm

Biesbroek, G. R., Klostermann, J. E. M, Termeer, C. J. A. M. \& Kabat, P. (2013). On the nature of barriers to climate change adaptation. Regional Environmental Change, 13 (5), 1119-1129. doi: 10.1007/s10113-013-0421-y

Blanco, H. P., McCarney, P., Parnell, S., Schmidt, M. \& Seto, K. C. (2011). The role of urban land in climate change. En C. Rosenzweig, S. A. Hammer, W. D. Solecki \& S. Mehrota (Eds.), Climate change and cities. First Assessment of the urban climate chance research network (pp. 217-248). Cambridge: Cambridge University Press.

Bulkeley, H. \& Betsill, M. M. (Eds.) (2003). Cities and climate change. Urban sustainability and global environmental governance. Oxon/New York: Routledge.

Burch, S. (2010). Transforming barriers into enablers of action on climate change: Insights from three municipal case studies in British Columbia, Canada. Global Environmental Change, 20 (2), 287-297. doi: 10.1016/j.gloenvcha.2009.11.009

Cetrángolo, O. (2007). Financiamiento municipal y combate contra la pobreza: Ejes de análisis [LC/L.2796-P]. Serie Medio Ambiente y Desarrollo 134. Santiago de Chile: Comisión Económica para América Latina y el Caribe (CEPAL). En http://www.cepal.org/es/ publicaciones/5676-financiamiento-municipal-combate-la-pobreza-ejes-analisis

Consejo Nacional del Ambiente (CONAm), Perú. (1999). Perú. Vulnerabilidad frente al cambio climático. Aproximaciones a la experiencia con el fenómeno El Niño. Lima: Autor. En http://bibliotecavirtual.minam.gob.pe/biam/handle/minam/723

Comisión Nacional del Medio Ambiente (conama), Chile. (2006). Estudio de la variabilidad climática en Chile para el siglo XXI. Informe final. Santiago: Autor. En http://dgf.uchile. cl/PRECIS/articles-39442_pdf_Estudio_texto.pdf

Comisión Nacional del Medio Ambiente (conama), Chile (2008). Plan de Acción Nacional de Cambio Climático. 2008-2012. Santiago: Autor.

Corfee-Morlot, J., Cochran, I., Hallegatte, S. \& Teasdale, P.-J. (2011). Multilevel risk governance and urban adaptation policy. Climatic Change, 104 (1): 169-197. doi: 10.1007/s10584-010-9980-9

Cortés, G., Schaller, S., Vargas, L., Rojas, M., García, L., Descalzi, A. \& McPhee, J. (2012). Assessment of the current climate and expected climate changes in the Metropolitan Region of Santiago de Chile (UFz-Report No. 03/2012). Leipzig: Helmholtz Centre for Environmental Research (UFz). En https://www.econstor.eu/ bitstream/10419/58274/1/717181324.pdf

Crabbé, P. \& Robin, M. (2006). Institutional adaptation of water resource infrastructures to climate change in Eastern Ontario. Climatic Change, 78 (1), 103-133. doi: $10.1007 /$ s10584-006-9087-5

Eisenack, K. \& Stecker, R. (2012). A framework for analyzing climate change adaptations as actions. Mitigation and Adaption Strategies for Global Change, 17 (3), 243-260. doi: 10.1007/s11027-011-9323-9

Fankhauser, S., Smith, J.B. \& Tol, R. S. J. (1999). Weathering climate change. Some simple rules to guide adaptation decisions. Ecological Economics, 30 (1), 67-78. doi: 10.1016/ S0921-8009(98)00117-7 
Fankhauser, S. (2009). A perspective paper on adaptation as a response to climate change. Copenhagen: Copenhagen Consensus Centre. En http://www.copenhagenconsensus. com/sites/default/files/pp_adaptation_fankhauser_v.3.0.pdf

Füssel, H.-M. (2007). Adaptation planning for climate change. Concepts, assessment approaches, and key lessons. Sustainable Science, 2 (2), 265-275. doi: 10.1007/ s11625-007-0032-y

Garreaud, S., René, D., Rutlland, C. \& José, A. (2006). Factores meteorológicos de la contaminación atmosférica. En R. G. E. Morales (Ed.), Contaminación atmosférica urbana. Episodios criticos de contaminación ambiental en la ciudad de Santiago (pp. 3554). Santiago: Editorial Universitaria.

Gawel, E., Heuson, C. \& Lehmann, P. (2012). Efficient public adaptation to climate changeAn investigation of drivers and barriers from a public choice perspective (UFz Discussion Paper No. 14/2012). Leipzig: Helmholtz Centre for Environmental Research (Ufz).

Hardoy, J. \& Lankao Romero, P. (2011). Latin American cities and climate change. Challenges and options to mitigation and adaptation responses. Current Opinion in Environmental Sustainability, 3 (3), 158-163. doi: 10.1016/j.cosust.2011.01.004

Heinrichs, D., Aggarwal, R., Barton, J., Bharucha, E., Butsch, C., Fragkias, M. \& Vogel, J. (2011). Adapting cities to climate change. Opportunities and constraints. En D. Hoornweg, M. Freire, M. J. Lee, P. Bhada-Tata \& B. Yuen (Eds.), Cities and climate change. Responding to an urgent agenda (pp. 193-224). Washington: Worldbank. En http://dx.doi.org/10.1596\%2F9780821384930_CH08

Heuson, C., Gawel, E., Gebhardt, O., Hansjürgens, B., Lehmann, P., Meyer, V. \& Schwarze, $\mathrm{R}$ (2012). Fundamental questions on the economics of climate adaptation - Outlines of a new research programme (UFz Report No. 05/2012). Leipzig: Helmholtz Centre for Environmental Research (UFZ).

Hunt, A. \& Watkiss, P. (2011). Climate change impacts and adaptation in cities: A review of the literature. Climatic Change, 104 (1), 13-49. En http://dx.doi.org/10.1007/ s10584-010-9975-6

Instituto Nacional de Estadística y Geografía (INEGI), México. (2010a). Sistema de Cuentas Nacionales de México. Producto Interno Bruto por Entidad Federativa, 2005-2009. Año base 2003. México, DF: Autor.

Instituto Nacional de Estadística y Geografía (Inegi), México. (2010b). Censo de Población y Vivienda 2010. México, DF: Autor. Consultado 12 de marzo de 2014. En http://www. censo2010.org.mx/.

Instituto Nacional de Estadística e Informática (IneI), Perú. (2010a). Producto Bruto Interno por Departamentos 2001-2009. Lima: Autor.

Instituto Nacional de Estadística e Informática (INeI), Perú. (2010b). Perú. Compendio Estadistico 2010. Lima: Autor. Consultado 12 de marzo de 2014. En http://www.inei. gob.pe/biblioineipub/bancopub/Est/Lib0982/compendio2010.html.

Instituto Nacional de Estadísticas (INE), Chile. (s/f). Proyecciones y estimaciones de población. Cifras de población estimadas a partir del Censo 2002 y estadísticas vitales, elaboradas a nivel nacional (1950-2050), regional, provincial y comunal (1990-2020). Santiago: Autor. Consultado 12 de marzo de 2014. En http://palma.ine.cl/demografia/menu/ EstadisticasDemograficas/proyecciones.aspx 
International Council for Local Environmental Initiatives (ICLEI). (2011). Financing the resilient city. A Demand driven approach to development, disaster risk reduction and climate adaptation - An ICLEI White Paper. Bonn: Autor. En https://www.environmentalfinance.com/content/research/financing-the-resilient-city.html

Joshi, M., Hawkins, E., Suttan, R., Lowe, J. \& Frame, D. (2011). Projections of when temperature change will exceed $2^{\circ} \mathrm{C}$ above pre-industrial levels. Nature Climate Change, 1, 407-412. doi: 10.1038/nclimate1261

Koch, I. C., Vogel, C. \& Patel, Z. (2007). Institutional dynamics and climate change adaptation in South Africa. Mitigation and Adaptation Strategies for Global Change, 12 (8), 1323 1339. doi: 10.1007/s11027-006-9054-5

London Climate Partnership. (2006). Adapting to climate change. Lessons for London. London: London Climate Partnership. En http://www.sfrpc.com/Climate\%20Change/9.pdf

Lehmann, P., Brenck, M., Gebhardt, O., Schaller, S. \& Süßbauer, E. (2015). Barriers and opportunities for urban adaptation planning: Analytical framework and evidence from cities in Latin America and Germany. Mitigation and Adaptation Strategies for Global Change, 20 (1), 75-97. doi: 10.1007/s11027-013-9480-0

Magee, N., Curtis, J. \& Wendler, G. (1999). The urban heat island effect at Fairbanks, Alaska. Theoretical and Applied Climatology, 64, 39-47. En http://akclimate.org/sites/default/ files//papers/Magee1999.pdf

Manning, M. R., Edmonds, J., Emori, S., Grubler, A., Hibbard, K., Joos, F., Kainuma, M., Keeling, R. F., Van Vuuren, D. P. (2010). Misrepresentation of the IPCC $\mathrm{CO}_{2}$ emission scenarios. Nature Geoscience, 3, 376-377. doi: 10.1038/ngeo880

Martínez López, B. (2011). Patrones de precipitación en el Valle de México, su evolución histórica y sus proyecciones. México, DF: Centro Virtual de Cambio Climático de la Ciudad de México.

McEvoy, D., Matczak, P., Banaszak, I. \& Chorynski, A. (2010). Framing adaptation to climaterelated extreme events. Mitigation and Adaptation Strategies for Global Change, 15 (7), 779-95. doi: 10.1007/s11027-010-9233-2

Measham T. G., Preston, B. L., Smith, T. F., Brooke, C., Gorddard, R., Withycombe, G. \& Morrison, C. (2011). Adapting to climate change through local municipal planning. Barriers and challenges. Mitigation and Adaptation Strategies for Global Change, 16 (8), 889-909. doi: 10.1007/s11027-011-9301-2

Municipalidad Metropolitana de Lima (MмL), Perú. (2012). La estrategia de adaptación de la Provincia de Lima al cambio climático. Lima: Autor.

Moser, S. C. \& Ekstrom, J. A. (2010). A framework to diagnose barriers to climate change adaptation. Proceedings of the National Academy of Sciences, 107 (51), 22026-22031. doi: $10.1073 /$ pnas. 1007887107

Müller, A. (2012). Areas at risk - Concept and methods for urban flood risk assessment. A case study of Santiago de Chile. Stuttgart: Franz Steiner Verlag.

Osberghaus, D., Dannenberg, A., Mennel, T. \& Sturm, B. (2010). The role of the government in adaptation to climate change. Environment and Planning C: Governance and Policy, 28 (5), 834-850. doi: 10.1068/c09179j 
Parry, M. L., Canziani, O. F., Palutikof, J. P., Van der Linden, P. J. \& Hanson, C. E. (2007). Cambio climático 2007: Impacto, adaptación y vulnerabilidad. Contribución del Grupo de Trabajo II al Cuarto Informe de Evaluación del IPCC. Cambridge, un: Cambridge University Press.

Rehner, J., Samaniego, J. \& Jordán, R. (2010). Regional Panorama: Latin America. Megacities and sustainability [LC/W.289]. Santiago: Comisión Económica para América Latina y el Caribe (CEPAL). En http://www.cepal.org/en/publications/3856-regional-panoramalatin-america-megacities-and-sustainability

Revi, A. (2008). Climate change risk: An adaptation and mitigation agenda for Indian cities. Environment and Urbanization, 20 (1), 207-229. doi: 10.1177/0956247808089157

Romero, H. \& Molina, M. (2008). Relación espacial entre tipos de usos y coberturas de suelos de islas de calor en Santiago de Chile. Anales Sociedad Chilena de Ciencias Geográficas, 1 (1), 223-230. En http://sochigeo.cl/2014/docs/Anales\%202007.pdf

Romero, P. (2010). Water in Mexico City. What will climate change bring to its history of water-related hazards and vulnerabilities? Environment and Urbanization, 22 (1), 157178. doi: $10.1177 / 0956247809362636$

Runhaar, H., Mees, H., Wardekker, A., Van der Sluijs, J. \& Driessen, P. P. J. (2012). Adaptation to climate change-related risks in Dutch urban areas: Stimuli and barriers. Regional Environmental Change, 12 (4), 777-790. doi: 10.1007/s10113-012-0292-7

Satterthwaite, D. (1997). Sustainable cities or cities that contribute to sustainable development? Urban Studies, 34 (10), 1667-1691. doi: 10.1080/0042098975394

Secretaría del Medio Ambiente (sMA), México. (2006). Estrategia local de acción climática del Distrito Federal. México, DF: Gobierno del Distrito Federal - Secretaría del Medio Ambiente (sMA). En http://centro.paot.org.mx/documentos/sma/ELACDF.pdf

Secretaría del Medio Ambiente (sma), México. (2008). Programa de Acción Climática de la Ciudad de México 2008-2012. México, DF: Gobierno del Distrito Federal - Secretaría del Medio Ambiente (sMA). En http://www.pgjdf.gob.mx/fedapur/DOCUMENTOS_ IMPORTANTES/programa\%20cambio\%20climatico\%20DF.pdf

Secretaría del Medio Ambiente (sma), México. (2011). Programa de Acción Climática de la Ciudad de México 2008-2012. Informe de Avances 2011. México: Gobierno del Distrito Federal - Secretaría del Medio Ambiente (sMA).

United Nations Development Programme (UNDP)/United Nations Environment Programme (unep). (2011). Mainstreaming Climate Change Adaptation into Development Planning: A Guide for Practitioners. Nairobi: Autores. En http://www.unep.org/pdf/ mainstreaming-cc-adaptation-web.pdf 